\title{
Orion Multi Purpose Crew Vehicle Environmental Control and Life Support Development Status
}

\author{
John F. Lewis ${ }^{1}$, Richard A. Barido ${ }^{2}$, and Cynthia D. Cross ${ }^{3}$ \\ NASA Johnson Space Center, Houston, Texas, 77058 \\ Robyn Carrasquillo ${ }^{4}$ \\ NASA Marshal Space Flight Center, Huntsville, Alabama, 35812 \\ and \\ George Edward Rains ${ }^{5}$ \\ Jacobs Engineering, Houston, Texas, 77058
}

\begin{abstract}
The Orion Multi Purpose Crew Vehicle (MPCV) is the first crew transport vehicle to be developed by the National Aeronautics and Space Administration (NASA) in the last thirty years. Orion is currently being developed to transport the crew safely from the Earth beyond Earth orbit. This year, the vehicle focused on building the Exploration Flight Test 1 (EFT1) vehicle to be launched in 2014. The development of the Orion Environmental Control and Life Support (ECLS) System, focused on the components which are on EFT1 which includes pressure control and active thermal control systems, is progressing through the design stage into manufacturing. Additional development work was done to keep the remaining component progressing towards implementation for a flight tests in 2017 and in 2020. This paper covers the Orion ECLS development from
\end{abstract}

April 2011 to April 2012.

\section{Introduction}

$\mathrm{D}$ evelopment of the Orion spacecraft has continued specifying and procuring components for the EFT1, while being pushed to fit within the design space of mass, power, and volume along with cost and schedule. This paper will encompass changes to the Orion Environmental Control and Life Support (ECLS) System since May 2011. This is the 7th in a series of ICES papers on Orion ECLS development status (ref 1-6).

This was a year of developing, designing, and building flight hardware and planning out the next missions past OFT1 for Orion.

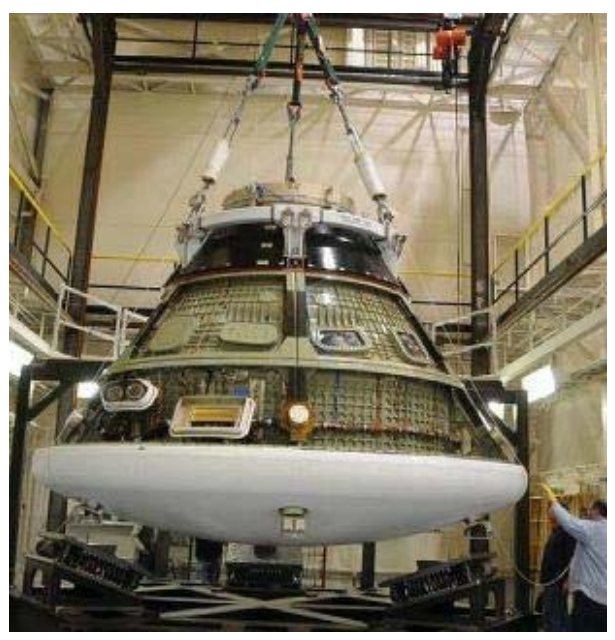

Figure 1: Orion Ground Test Article

\footnotetext{
${ }^{1}$ Orion ECLS System Manager, Crew and Thermal Division, EC6.

${ }^{2}$ Orion ECLS ARS Subsystem Manager, Crew and Thermal Division, EC6.

${ }^{3}$ Orion ECLS ATCS Subsystem Manager, Crew and Thermal Division, EC6.

${ }^{4}$ Orion Crew and Thermal FAM, Systems Development, Integration, and Test, ES62. AIAA Member

${ }^{5}$ Orion ECLS PSC Subsystem Manager, Crew and Thermal Division, EC6.
} 


\section{A. MPCV Development Manifest}

The Orion Program is developing the flight test manifest to coincide with the available Space Launch System (SLS) development launch schedule and to fit within the predicted budget. There are four flights, EFT1, AA2, EM1, and EM2.

EFT1 (formerly known as Orion Flight Test 1 - OFT1) will tests out the thermal protection system heat shield and parachute recovery systems. This flight launches an Orion capsule without crew for two orbits and performs a high speed entry close to Lunar return velocities. On EFT1, ECLS will have Active Thermal Control to provide cooling to the flight avionics and Cabin Pressure Relief to protect the sealed volume. EFT1 launch is scheduled in XXX 2014 on a Delta IV heavy from Launch Complex 37A at Cape Canaveral.

AA2 will be a launch abort test at maximum dynamic pressure. The flight launches and Orion capsule with now crew to an altitude of 70,000 ft (21,336 m) where the launch abort system will pull the capsule from the launch vehicle to safety. Since the same capsule from EFT1 is planned to be used for AA2, the ECLS system will be the same as EFT1. It is still to be determined what ECLS hardware will be active for that flight depending on the avionics configuration and the duration of the mission. AA2 launch is scheduled in ???? on a Peacekeeper missile from Cape Canaveral.

EM1 will launch an unmanned Orion on a free return flight around the Moon launched on the first flight of the SLS vehicle. On this flight ECLS will add the external thermal loop with radiators to the EFT1 configuration to dissipate heat for the extended duration mission. EM1 launch is planned for 2017 from Kennedy Spaceflight Center.

EM2 will be the first crewed launch of Orion and is planned to orbit the Moon and return. On this flight, there will be a full ECLS system including atmospheric revitalization, pressure control, water storage, waste collection and emergency response. EM2 is planned for launch on the SLS from Kennedy Spaceflight Center in 2020.

\section{B. Affordability Efforts}

To fit the funding made available to Orion, efforts are underway at the Orion program level and in the ECLS System to reduce costs. At the program level, Orion is looking at options like protoqualing components to avoid the cost of building additional qualification hardware by flying the hardware that you use to qualify the design, and deferring human systems hardware like ECLS and flight crew equipment until EM2.

Within ECLS there was activity between Lockheed Martin and NASA to determine how to reduce the cost of the ECLS system. Lockheed is limited to a minimal efforts of any part of Orion past EFT1. To bridge this gap, NASA ECLS has taken on tasks in key areas like development of the suit loop components, integrated testing, and requirements refinement. This activity is to continue the work that Lockheed cannot perform and to have a cleaner more specific set of requirements that will be lower cost for Lockheed to build and verify. NASA and Lockheed are also performing trades on Active Thermal Control, Atmospheric Revitalization, and Pressure Control configurations to produce a lower cost approach. The team is also working with Hamilton Sundstrand to see if there are any manufacturing efficiencies like using common actuators, heritage designs, or reduction of overlapping standards and requirements between NASA Lockheed and Hamilton.

\section{Ambient Pressure Integrated Suit Test (APIST)}

The integration between the crew pressure suit and the life support system is hard to model due to flows and pressure drops being affected by crew motion and the changing volume of the suit. The NASA Orion ECLS team used development hardware of the amine swing bed for $\mathrm{CO} 2$ and humidity control, suit loop fan, trace contaminant control, and development suits to test in an integrated configuration based on the Orion Suit Loop. Since the development hardware is not rated for elevated oxygen levels, the test was performed at ambient pressures and a suit delta pressure from 0.5 psid to 2.0 psid. The test showed that the pressure changes in the suit from swing bed cycling or suit movement were not an issue for the test subjects. Also, acoustics and suit humidity levels were acceptable. Future testing in the upcoming years is planned at 10.2 psia and vacuum that will additionally integrate components rated for elevated oxygen, a development suit pressure regulator and a development liquid cooling loop. Reference APIST Paper here 


\section{System Status}

\section{A. Active Thermal Control}

EFT1 Configuration: The Active Thermal Control Subsystem (ATCS) configuration includes the pump package of four pumps (redundant pumps on each of two loops), accumulators, Service Module isolation valves, ground support heat exchanger, eleven cold plates, two ammonia tanks, and two ammonia boilers. The ATCS uses a 50\% propylene glycol in water coolant for a thermal transport to provide cooling for vehicle avionics. Cooling is provided from pre-launch using the ground support heat exchanger, through launch, orbit, re-entry, and landing using the ammonia boilers. Radiators are being built, but do not include the flow tube network since they are not needed for this flight test. Depending only on consumable ammonia for heat dissipation, EFT1 has a limited amount of cooling.

Ammonia boiler under performance: Testing of two development ammonia boilers were not able to achieve the performance required for the fully operational Orion system like what will be used on EM2. The challenges of cooling a fluid like propylene glycol is challenging due to the increase in viscosity at colder temperatures where the issue in the tests. While the performance will be adequate for EFT1, further development will be required to meet the EM2 loads.

Use of Shuttle GSE Heat Exchanger: Additional testing is in work to test the Shuttle GSE Hx with the 50\% Propylene glycol and R-134a coolants that will be used on the EFT1 vehicle. Issues with taking existing certified hardware from another flight program and certifying the hardware for use on EFT1 became an issue that had to be worked. These issues are covered in the paper REF:

Active Thermal Architecture Trade: Both NASA and Lockheed Martin together are trading alternate architectures to determine the optimum configuration for the possible missions for the MPCV. The trade is looking at single fluid, heat pipe radiators, and the baseline configuration and how well the support Earth orbital, Lunar orbital, and deep space missions. The results could lead to the EM1 mission using this alternate architecture.

Liquid Cooling Garment (LCG) Loop Developments: NASA is developing a venting gas trap for the liquid cooling garment. During disconnects and reconnects of the suit to the vehicle systems, gas is entrained into the cooling water and has to be removed to assure pump operation. Baseline design uses a non-venting gas trap that can only separate a fixed amount of gas. A development venting gas trap is being built and will be tested in the following year (REF: IS THERE A GRANT PAPER?)

\section{B. Air Revitalization}

EFT1 Configuration: Since there are no crew for the EFT1 flight test, no air revitalization or suit support in the EFT1 vehicle

Development Suit Loop Fan: For the APIST test, NASA utilized the development fan that Hamilton Sunstrand produced to develop fan curves. The fan had similar performance to flight but was manufactured using a stereolithography (SLA) approach. NASA is pursuing development of a higher fidelity suit loop fan that incorporates the flight materials and motor. This will incorporate the ceramic "canning" of the motor required for oxygen safety and is a development risk. This fan will be used in the future integrated suit loop tests.

CO2 And Moisture Removal Assembly (CAMRAS) Flight Test on ISS: NASA launched one of the development CAMRAS unit coupled with a moisture recuperator to be tested on the International Space Station (ISS). The unit has been launched and installed. The team is still working out integration issues to get the unit started, so testing will be conducted next year.

SBAR and Alternate TCCS Designs: NASA is designing alternate CO2/Humidity removal approach using zeolite as opposed to the baseline of amine to see if a simpler and more affordable system. NASA is also looking at alternate materials for the trace contamination control beds in Orion to improve on performance, weight and volume. IS THERE A PAPER HERE 
Orion Air Monitor (OAM) common with ISS Major Constituent Analyzer (MCA): Hamilton Sunstrand is developing an approach to produce a qualified air monitor design to monitor oxygen, carbon dioxide, nitrogen, humidity, hydrogen, and methane, that could be used for the MPCV and also serve as an upgrade to the ISS MCA. NASA is working to see if this can be supported programmatically. IS THERE A PAPER HERE

\section{Pressure Control}

EFT1 Configuration: The Pressure Control Subsystem (PCS) configuration includes a positive pressure relief valve (PPRV) and a negative pressure relief valve (NPRV). The components to provide oxygen and nitrogen to the cabin have been deferred from EFT1.

Use of ISS Positive Pressure Relief Assembly (PPRA): Some additional vibration testing may be required since the ISS PPRA qualification do not completely cover the Orion EFT1 environments. Issues with taking existing certified hardware from another flight program and certifying the hardware for use on EFT1 became an issue that had to be worked. These issues are covered in the paper REF:

Negative Pressure Relief Valve (NPRV) Dual Isolation: It was decided that the NPRV needed to be able to seal against water after a failure in the NPRV. The negative pressure relief valve protects the cabin from structural collapse if the internal pressure is lower than 1 psid $(6.9 \mathrm{KPa})$ from the external pressure due to a cabin leak. There are two nominal landing orientations, one with the heat shield in the down and one with the docking tunnel down. In the latter configuration, the water pressure would be high enough to allow water to come into the vehicle. The NPRV is being designed with a secondary isolation feature that would protect for this.

Use of Propulsion pressure components for ECLS pressure control: Lockheed is investigating use of pressure supply regulators, check valves, and isolation valves certified for the propulsion system to be used regulate and control the PCS for Orion ECLS. This would be a cost savings to avoid multiple component certifications. Work is still ahead to see if the propulsion system components are the right performance to meet the PCS needs, and if they can be made to be compatible to work with high pressure oxygen.

\section{Emergency Response}

EFT1 Configuration: Since there are no crew for the EFT1 flight test, no emergency response in the EFT1 vehicle.

Nantube based E-nose Sensor Development: Work is underway to develop a nanotube based e-nose sensor to detect ammonia and hydrazine for post landing contaminants, and carbon monoxide and acid gases for post fire contaminants. Development units will be tested against real fire environments to better understand the viability of the approach.

\section{E. Potable Water \& Waste Management}

EFT1 Configuration: Since there are no crewmembers or active thermal control sublimators that require water for the EFT1 flight test, no potable water or waste management in the EFT1 vehicle.

\section{Conclusion}

Design and manufacture of the ECLS hardware is well underway to meet the EFT1 mission. Plans are being made for the following Orion flights that will ultimately put crews out past low Earth orbit. To meet that end focused testing on high risk ECLS areas which are not part of the EFT1 mission are also being performed. 


\section{References}

${ }^{1}$ Lewis, J., “The Crew Exploration Vehicle Environmental Control and Life Support Development Status - 2006” SAE Technical Paper Series 2006-01-2011, 36th International Conference on Environmental Systems, Newport, VA July 2006.

${ }^{2}$ Lewis, J., Barido, R., Carrasquillo, R., Cross, C., Tuan, G.,"The Crew Exploration Vehicle Environmental Control and Life Support Development Status - 2007” SAE Technical Paper Series 2007-01-3044, 37th International Conference on Environmental Systems, Chicago, IL July 2007.

${ }^{3}$ Lewis, J., Barido, R., Carrasquillo, R., Cross, C., Tuan, G.,"The Crew Exploration Vehicle Environmental Control and Life Support Development Status - 2008” SAE Technical Paper Series 2008-01-2079, 38th International Conference on Environmental Systems, San Francisco, CA July 2008.

${ }^{4}$ Lewis, J., Barido, R., Carrasquillo, R., Cross, C., Tuan, G., “The Crew Exploration Vehicle Environmental Control and Life Support Development Status - 2009" SAE Technical Paper Series 2009-01-2457, 39th International Conference on Environmental Systems, Savannah, GA July 2009.

${ }^{5}$ Lewis, J., Barido, R., Carrasquillo, R., Cross, C., Tuan, G.,"The Crew Exploration Vehicle Environmental Control and Life Support Development Status - 2010” AIAA Technical Paper Series 763172R1, 40th International Conference on Environmental Systems, Barcelona Spain July 2010.

${ }^{6}$ Lewis, J., Barido, R., Carrasquillo, R., Cross, C., George E. Rains.,“The Crew Exploration Vehicle Environmental Control and Life Support Development Status - 2011” AIAA Technical Paper Series ???????, 41th International Conference on Environmental Systems, Portland, Oregon July 2011. 